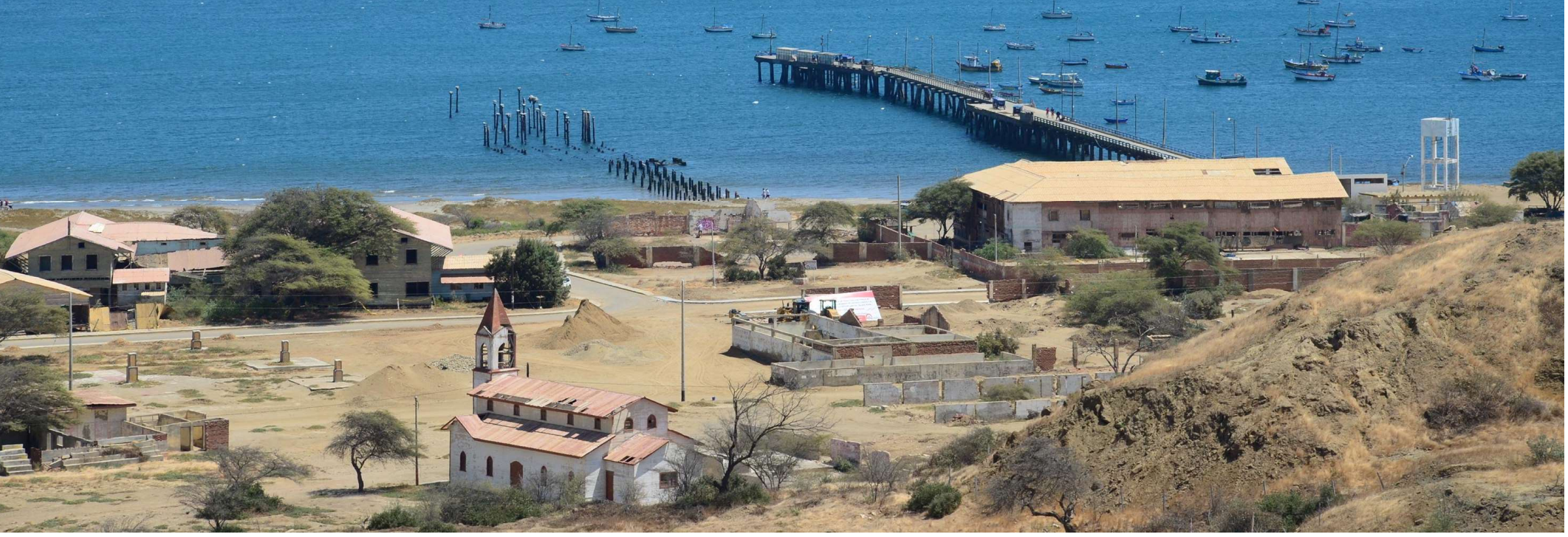

\title{
EXAMINANDO LOS
}

CRUDOS DETALLES

Auditorías gubernamentales de los costos de proyecto del petróleo y gas para maximizar la recaudación fiscal

Resumen Ejecutivo 
El sector petrolero ofrece a los gobiernos un alto potencial de ingresos que podrían invertirse en la reducción de la pobreza e inequidad, pero esos ingresos primeramente deben ser recaudados. Los impuestos se gravan sobre las ganancias; sin embargo, las empresas pueden intentar reducirlos mediante la deducción de costos no elegibles o extraordinarios, los cuales a menudo se pagan a empresas asociadas. El derecho a llevar a cabo una auditoría de costos es la herramienta fundamental de los gobiernos para combatir la exageración de los costos del petróleo, pero la información existente para determinar si los gobiernos ejercen este derecho de manera efectiva, es limitada. Las prácticas de auditoría en Ghana, Kenia y Perú sugieren que los gobiernos enfrentan importantes desafíos. Oxfam propone recomendaciones para abordar estos desafíos y garantizar que los gobiernos recauden los impuestos que se les deben de la explotación de sus recursos petroleros finitos y no renovables. 
Este documento fue redactado por Alexandra Readhead, Daniel Mulé y Anton Op de Beke. Los autores agradecen a todas las personas que contribuyeron en la elaboración de este reporte, incluyendo aquellos que proporcionaron sus valiosas aportaciones mediante entrevistas y correspondencia escrita. Los autores agradecen especialmente, la franqueza, cooperación y el tiempo que los representantes de los gobiernos de Ghana, Kenia y Perú destinaron a este proyecto. También agradecemos a Ben Boakye, Jack Calder, Humberto Campodónico, Thomas Lassourd, Charles McPherson, Rob Veltri y Charles Wanguhu por sus excelentes aportaciones. Extendemos un especial agradecimiento por su colaboración, a nuestros colegas de Oxfam Francis Agbere, Miguel Lévano, Gilbert Makore, Armando Mendoza y Davis Osoro por su dedicación para garantizar la calidad de la investigación de los estudios de caso. Manifestamos especial agradecimiento a la contribución de otros compañeros en Oxfam, entre ellos Alejandra Alayza, Alex Ampaabeng, Andrew Bogrand, Kathleen Brophy, Nathan Coplin, Nadia Daar, Nick Galasso, Christian Hallum, Richard HatoKuevor, Joy Kyriacou, Max Lawson, Robert Maganga, Kevin May, Abdul Karim Mohammed, Joy Ndubai, Oli Pearce, Marta Pieri, Quentin Parrinello, María Ramos, Radhika Sarin, Claudia Sánchez, Robert Silverman, Kate Stanley e lan Thomson. También agradecemos a quienes hicieron posible la publicación de este reporte, en particular a lan Gary, Emily Greenspan e Isabel Munilla por el reconocimiento oportuno de la importancia de esta temática y su apoyo inquebrantable, y a James Morrissey y Laurel Pegorsch por su incansable apoyo y constante atención al detalle. Este reporte también fue posible gracias al apoyo de las Fundaciones Ford y William and Flora Hewlett. Les agradecemos por seguir creyendo en nuestra labor para promover la justicia fiscal y responsabilidad ciudadana, y por fomentar las mejores prácticas en la gobernanza de los sectores del petróleo, gas y minería.

Para más información o realizar comentarios sobre este informe, póngase en contacto con: advocacy@oxfaminternational.org

Esta publicación está sujeta a copyright pero el texto puede ser utilizado libremente para la incidencia política y campañas, así como en el ámbito de la educación y de la investigación, siempre y cuando se indique la fuente de forma completa. El titular del copyright solicita que cualquier uso de su obra le sea comunicado con el objeto de evaluar su impacto. La reproducción del texto en otras circunstancias, o su uso en otras publicaciones, así como en traducciones o adaptaciones, podrá hacerse después de haber obtenido permiso y puede requerir el pago de una tasa. Debe ponerse en contacto con policyandpractice@oxfam.org.uk.

La información que hay en esta publicación se encuentra actualizada al momento en que se envió a la imprenta.

Publicado por Oxfam GB para Oxfam International con ISBN 978-1-78748-361-3 en noviembre 2018.

DOI: $10.21201 / 2018.3590$

Oxfam GB, Oxfam House, John Smith Drive, Cowley, Oxford, OX4 2JY, UK.

Foto de portada: Una bomba de extracción petrolera en la costa de Perú, en las afueras de Lobitos, Talara, Piura (Rafael Storch). (Rafael Storch). (Rafael Storch). 
Una función vital del gobierno es la recaudación de ingresos a través de impuestos y otras vías. Los gobiernos requieren ingresos para financiar gastos tales como inversiones en infraestructura y servicios sociales, entre los cuales se encuentran la salud y la educación. La tributación progresiva y el gasto público progresivo son fundamentales para combatir la desigualdad y erradicar la pobreza. ${ }^{1}$ Pese al esfuerzo concertado en varios países en desarrollo, la recaudación de ingresos sigue siendo demasiado baja. El Banco Africano de Desarrollo declara que para que los países africanos puedan financiar las necesidades de infraestructura y desarrollo humano en el continente, necesitarán incrementar la proporción promedio de impuestos al producto interno bruto (PIB) a un 25 por ciento, ${ }^{2}$ arriba del 19.2 por ciento actual. ${ }^{3}$

Las industrias extractivas - petróleo, gas y minería - representan una gran oportunidad para movilizar importantes ingresos fiscales nacionales que se pueden emplear para reducir la brecha entre ricos y pobres. En particular, el sector petrolero a menudo genera abundantes ganancias y los gobiernos, como los custodios de este recurso, tienen derecho a la mayor parte del pastel. Incluso cuando las empresas de petróleo y gas ya contribuyen considerablemente a los ingresos del gobierno, es posible que deban contribuir aún más. Sin embargo, en la práctica no es tan fácil que los gobiernos de los países en desarrollo administren adecuadamente sus regímenes fiscales petroleros y recauden todo lo que se les debe. Las autoridades tributarias pueden carecer de los instrumentos legales y regulatorios necesarios, información o conocimiento técnico; además, los incentivos contrapuestos o las presiones políticas pueden complicar su mandato de maximizar la recaudación de ingresos.

Los desembolsos inflados de las empresas representan una grave amenaza para los ingresos del gobierno provenientes del petróleo y gas. Mientras mayores sean los costos que reportan las empresas, menores serán las ganancias gravables; esto significa menos ingresos para el gobierno. Los países en desarrollo tienen más que perder cuando se presentan costos exagerados, debido a su excesiva dependencia de los impuestos sobre sociedades. ${ }^{4}$ Las empresas también pueden deliberadamente intentar evadir o eludir impuestos. La evasión es el uso de métodos legales (a diferencias de los métodos ilegales) para minimizar el adeudo de impuestos sobre la renta de las corporaciones multinacionales (CMNs). El reciente escándalo fiscal de una empresa petrolera en Australia confirma que cuando las empresas obtienen bienes, servicios y deudas de otras empresas relacionadas bajo la misma CMN, existe el riesgo de que se exageren los costos, con el propósito de reducir sus propias ganancias y que las mismas se transfieran al extranjero. ${ }^{5}$

Oxfam y sus aliados promueven mejorar la gobernanza fiscal de las industrias extractivas, mediante una mayor transparencia y supervisión. ${ }^{6}$ Así como lo demostró Oxfam en su proyecto de evaluación de riesgos 4

\author{
Las industrias \\ extractivas - petróleo, \\ gas y minería- \\ representan una gran \\ oportunidad para \\ movilizar importantes \\ ingresos fiscales \\ nacionales que se \\ pueden emplear para \\ reducir la brecha entre \\ ricos y pobres.
}

Las empresas también pueden deliberadamente intentar evadir o eludir impuestos. La evasión es el uso de métodos legales (a diferencia de los métodos ilegales) para minimizar la deuda fiscal de las corporaciones multinacionales (CMNs). 
para los ingresos, ${ }^{7}$ un área crítica que requiere fortalecimiento es la efectividad del gobierno en las auditorías fiscales.

Los países productores de petróleo deben asegurarse de retener una proporción adecuada del valor de su petróleo y gas sí quieren lograr sus objetivos de movilización de ingresos fiscales nacionales; de lo contrario, podrían perder millones de dólares. En dos ejemplos distintos, los auditores descubrieron que las empresas de petróleo y gas habían exagerado sus costos en 127 millones en la República del Congo y en 81 millones en Uganda. Como resultado se registraron pérdidas de ingresos significativas: \$ 63.5 millones en Congo y \$ 24 millones en Uganda (Véase la parte de la "Introducción"). Este estudio identifica seis desafíos recurrentes en Ghana, Kenia, y Perú para llevar a cabo una auditoría de costos efectiva, que podrían ser pertinente a la experiencia de otros países que producen petróleo:

- Las leyes que determinan el manejo y la elegibilidad de los costos del petróleo son inadecuadas y la implementación de cambios puede ser complicada. Una auditoría sólo es efectiva en la medida en que la ley obligue su cumplimiento. Las lagunas fiscales y los costos que son propensos a ser manipulados pueden limitar la capacidad del auditor para proteger los ingresos del gobierno.

- La fragmentación institucional entorpece la administración efectiva de los ingresos. Los derechos de auditoría de costos a menudo están divididos entre varias dependencias gubernamentales, las cuales tienen conflictos en su mandato. De tal manera que una coordinación deficiente inevitablemente conduce a la duplicación de esfuerzos y a una incertidumbre para los inversionistas en relación al cálculo final del ingreso bruto.

- Los auditores carecen de conocimientos técnicos y especializados en el sector. La falta de una evaluación periódica de riesgos en el sector petrolero refleja la limitada comprensión por parte de las autoridades fiscales sobre las particularidades de la industria. A pesar de esta brecha, ni Ghana ni Kenia han empleado la opción reservada para ellos en varios de sus acuerdos petroleros para subcontratar —parcialmente a expensas del contratista - auditores externos para llevar a cabo una inspección de las cuentas de las empresas.

- Es difícil recabar información para tener un análisis comparativo para los costos del petróleo. Los requisitos de presentación de informes son insuficientes o poco claros, lo cual impide que los gobiernos accedan a determinada información de las empresas. No obstante, a nivel mundial se han puesto en marcha esfuerzos para ampliar el conjunto de datos disponibles, especialmente en relación con los costos del petróleo.

- Las auditorías se llevan a cabo demasiado tarde. Hay una tendencia a priorizar las auditorías sólo cuando el petróleo está ya en flujo, mucho tiempo después de que se ha comenzado el desarrollo de la explotación petrolera. Para entonces, es posible que el derecho que tiene el gobierno a realizar una auditoría haya ya expirado, junto con la obligación jurídica de la empresa de guardar sus registros. 
- Hay falta de transparencia y rendición de cuentas pública en las auditorías de costos. La información que está disponible al público no proporciona suficientes bases para evaluar cómo, y si los gobiernos están ejerciendo su derecho a la auditoría. Y al menos que un caso termine en los tribunales, los ciudadanos no tienen ni idea de si su gobierno está auditando los costos o cual puede ser su impacto. Las Entidades Fiscalizadoras Superiores (EFS), legislaturas nacionales, comisiones independientes, así como los grupos de múltiples partes interesadas que conforman la Iniciativa para la Transparencia de las Industrias de Extractivas (EITI por sus siglas en inglés), todos ellos pudieran potencialmente evaluar las prácticas de auditoría de costos, pero normalmente no lo hacen. 


\section{RECOMENDACIONES}

En base al análisis de estos frecuentes desafíos, este reporte presenta una serie de recomendaciones que pudieran ayudar a Ghana, Kenia, Perú y a otros países productores de petróleo que estén enfrentando desafíos similares para limitar los riesgos de la exageración de costos mediante el uso de prácticas de auditoría efectivas.

1. LEYES: Analizar y fortalecer los controles jurídicos de los costos del petróleo. Crear cuidadosamente leyes que determinen los criterios y el manejo de los costos, para que así los auditores cuenten con las herramientas legales necesarias para proteger los ingresos del gobierno.

2. COORDINACIÓN: Definir con claridad cuáles son las dependencias gubernamentales responsables de las auditorías de costos y de fortalecer la coordinación interinstitucional de la gestión de los ingresos del petróleo. Los mecanismos de coordinación deben facilitar el intercambio de información y conocimiento técnico a través de las agencias gubernamentales, incluyendo asimismo a las empresas petroleras nacionales.

3. CAPACIDAD: Desarrollar los conocimiento técnicos y especializados en el sector para detectar y mitigar la exageración de costos en el sector petrolero. El abordar las auditorias con un enfoque en base a los riesgos y al acceso a la información, es especialmente importante para los países de recursos limitados, ya que asegurar que los escasos recursos humanos y financieros sean invertidos de forma sensata.

4. INFORMACIÓN: Adoptar las medidas necesarias para mejorar la información disponible y así verificar y estimar los costos del petróleo. Se deben explorar vías innovadoras para que los productores de petróleo intercambien información de forma anónima sobre los costos, con el propósito de contar con mayores datos de referencia.

5. MARGEN DE TIEMPO: Asegurar que los plazos previstos para las auditorías, y requisitos para guardar los registros sean lo suficientemente largos, así como también que los costos sean auditados tan pronto como sean efectuados. Los gobiernos deben de llevar acabo las auditorias tan pronto y como las empresas petroleras comiencen sus actividades, en lugar de esperar a que los ingresos comiencen a fluir, ya que para entonces puede que los derechos a la auditoria ya hayan expirado.

6. RENDICIÓN DE CUENTAS: Divulgar públicamente las actividades de auditoría y sus resultados, así como fortalecer la capacidad de supervisión de los actores para monitorear el uso de los derechos de auditoría de los gobiernos. Un proceso de auditoria transparente y responsable es un requisito previo para tener una auditoría de costos efectiva; sin acatar esto, es imposible determinar si los gobiernos están cumpliendo con su compromiso de proteger los ingresos provenientes del petróleo. 


\section{Resumen de Recomendaciones por Actor}

\section{Los gobiernos}

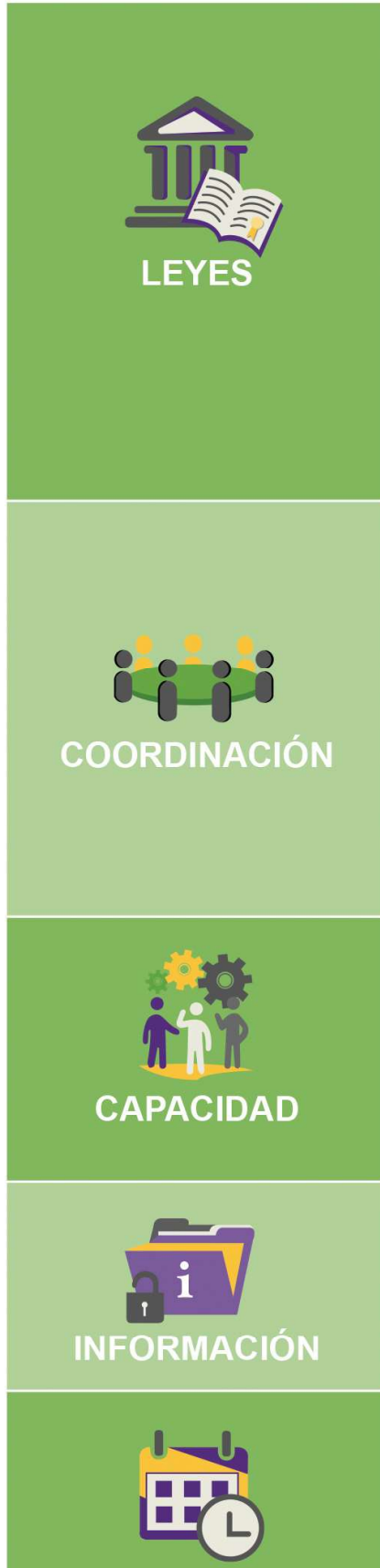

MARGEN DE TIEMPO

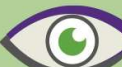

RENDICION DE

CUENTAS
- Dar una definición clara de los fondos de disponibilidad limitada dentro de la ley.

- Publicar una guía para los contribuyentes sobre los elementos fiscales que puedan ser malinterpretados.

- Adoptar e implementar reglas más enérgicas de precios de transferencia, además de explorar normas alternativas de políticas fiscales que puedan facilitar su gestión.

- Considere cuidadosamente la elegibilidad de los costos para la recuperación de costos y / o deducciones de impuestos.

- Asegurarse de que las cláusulas de estabilización fiscal sean limitadas en tiempo y alcance.

- Asignar la responsabilidad de la auditoría de costos a una sola agencia o bien formalizar la cooperación entre agencias.

- Fomentar una supervisión sólida por parte de las TSC, pero no la auditoría directa.

- Asegurar que las empresas petroleras nacionales (EPNs) compartan los resultados de las auditorias de la empresa en participación con las agencias de auditoria de costos.

- Promulgar una ley para crear un comité interinstitucional con un presupuesto estable.

- Asignar un número adecuado de personal para las auditorías de costos del petróleo.

- Desarrollar conocimientos especializados de la industria petrolera.

- Considere solicitar auditores externos independientes para realizar auditorías de costos.

- Hacer cumplir los requisitos de informes claros y estándar para su uso en la evaluación de riesgos.

- Adoptar las normas de documentación de precios de transferencia, incluyendo un requisito para archivos internos y maestros.

- Asegúrese de que el límite de tiempo para realizar una auditoría no sea imposiblemente corto.

- La evaluación de costos comienzan a realizarse incluso antes de que los ingresos comiencen a fluir.

- Aprobación previa de los gastos de petróleo presupuestados de las compañías de petróleo y gas.

- Publicación de auditorías y de un informe anual que describa el uso de los derechos y resultados de la auditoría de costos.

- Desarrollar el conocimiento de la industria de las TSC para un monitoreo más efectivo.

- Publicación de auditorías y de un informe anual que describa el uso de los derechos y resultados de la auditoría de costos. 


\section{La sociedad civil}

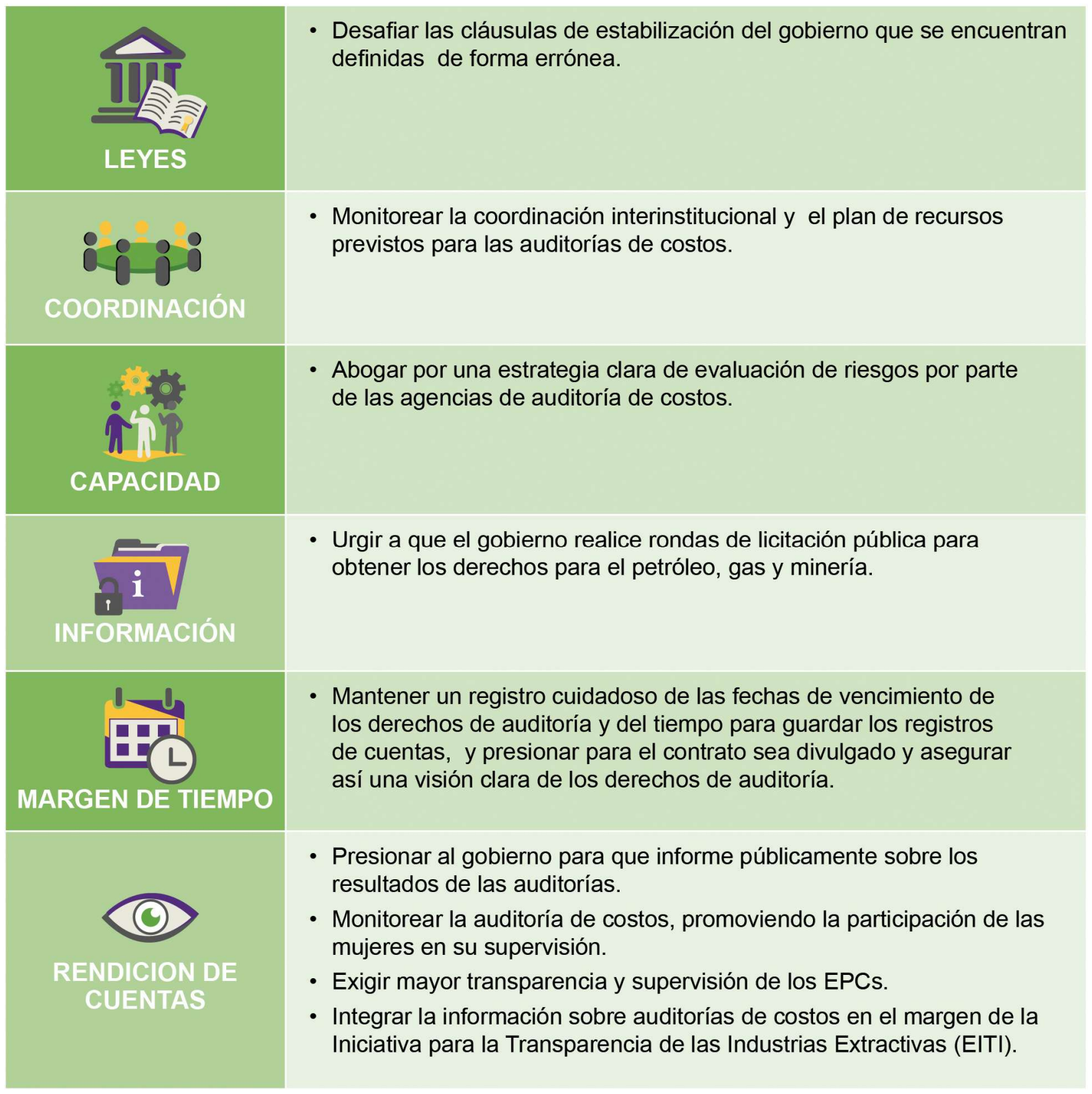




\section{La cooperación internacional}

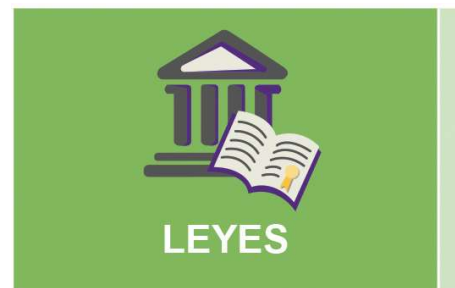

- Oponerse a las cláusulas gubernamentales de estabilización que estén poco o mal definidas.

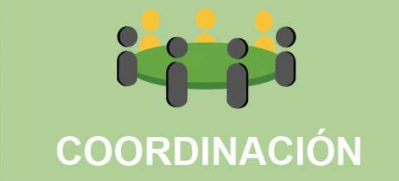

- Brindar apoyo técnico y financiero a los comités interinstitucionales.

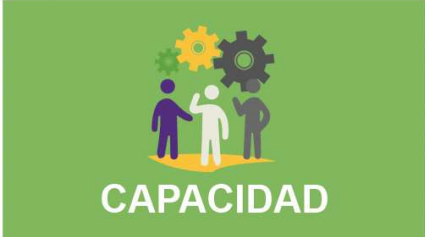

- Apoyar las solicitudes del gobierno para el desarrollo de capacidades y subcontratación externa para solo algunos elementos de la auditoría (co-sourcing en inglés).

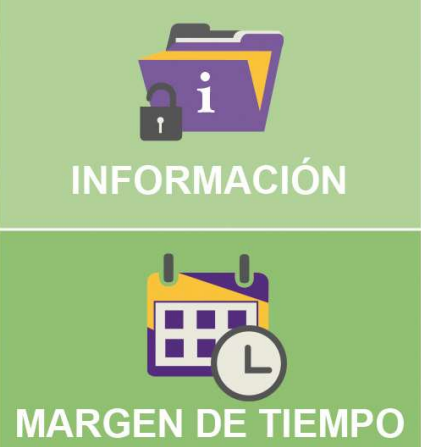

- Apoyar el acceso a las bases de datos comerciales e intercambio de información.

- Mantener un registro cuidadoso de las fechas de vencimiento de los derechos de auditoría y de los registros de cuentas.

- Abogar por auditorías de costos rigurosas y la publicación de las auditorias, así como la divulgación y supervisión del proceso y de los resultados de la auditoría.

- Recopilar datos cuantitativos sobre el uso de los derechos de auditoría de costos. 
Las empresas petroleras

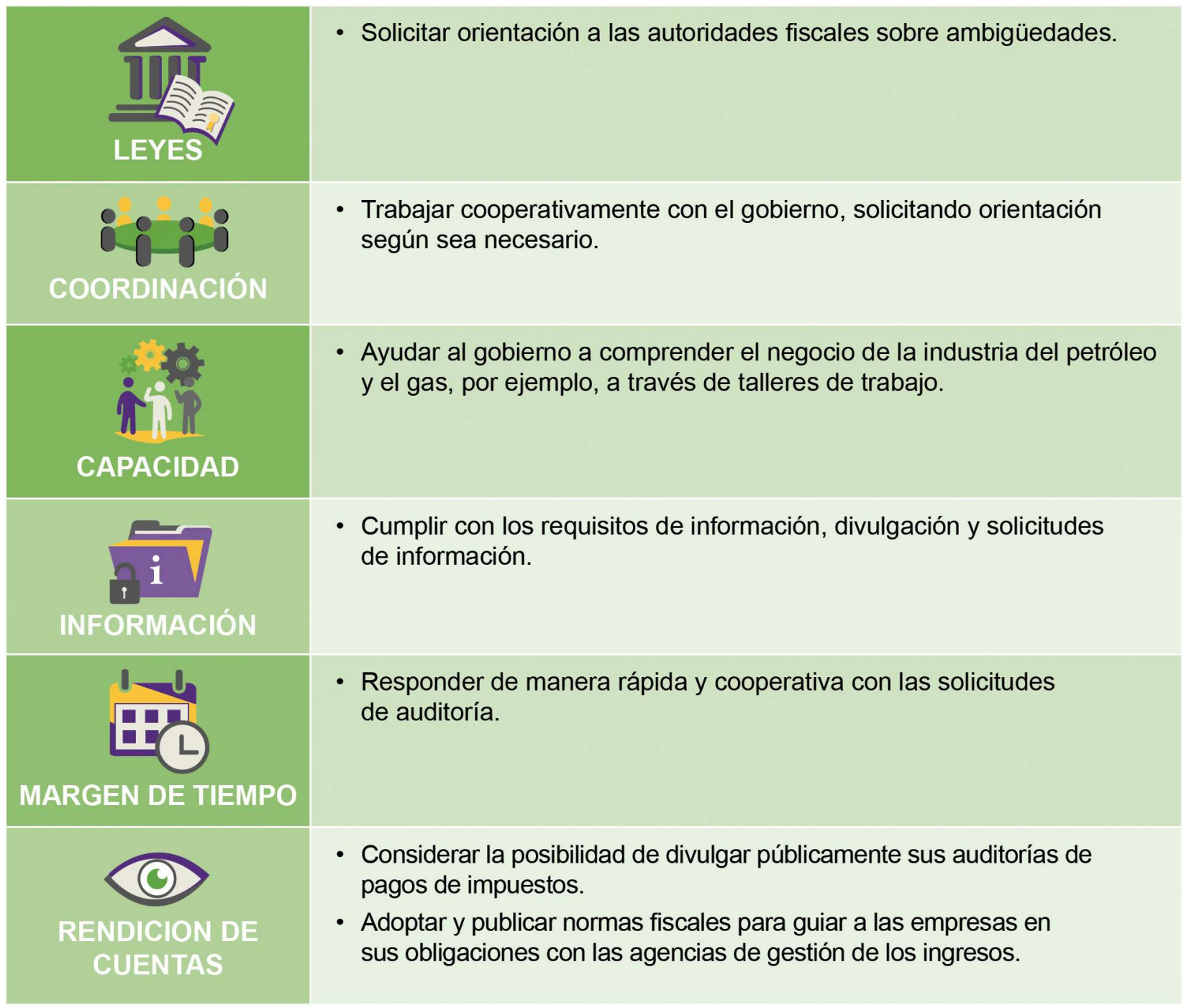




\section{NOTAS}

${ }^{1}$ Ver en general, Oxfam, The Commitment to Reducing Inequality Index 2018, October 8, 2018, https://policypractice.oxfamamerica.org/publications/the-commitment-to-reducing-inequality-index-2018/

2 African Development Bank. (2018). African Economic Outlook 2018, https://www.afdb.org/fileadmin/uploads/afdb/Documents/Publications/African Economic Outlook 2018 EN.pdf.

${ }^{3}$ OECD (Organisation for Economic Co-operation and Development), Revenue Statistics in Africa, 2017, https://www.oecd.org/tax/tax-policy/revenue-statistics-africa-2017-infographic.pdf. IMF, Regional Economic Outlook for Sub-Saharan Africa (Washington, DC, 2017), https://www.imf.org/ /media/Files/Publications/REO/AFR/2017/May/pdf/sreo0517.ashx. Este reporte cita el 17.0 por ciento como la proporción promedio impuesto/PIB de la región en 2017 y concluye que para la mayoría de los países es factible un incremento de 3-5 puntos. IMF, Regional Economic Outlook: Domestic Revenue Mobilization and Private Investment (Washington, DC, 2018), https://www.imf.org/ /media/Files/Publications/REO/AFR/2018/May/pdf/sreo0518.ashx?la=en

${ }^{4}$ UNCTAD, World Investment Report 2015: Reforming International Investment Governance (Geneva, 2015), p. 182, https://unctad.org/en/PublicationsLibrary/wir2015 en.pdf

${ }^{5}$ P. Wells, "Chevron Loses Landmark Tax Case on Transfer Pricing," Financial Times, April 20, 2017, https://www.ft.com/content/9eaef50e-264f-11e7-a34a-538b4cb30025

${ }^{6}$ El trabajo de Oxfram en las industrias de la extracción abarca una variedad de contenidos temáticos, entre ellos los impuestos, la transparencia y el consentimiento de la comunidad: "Oxfam advocates for just government policies and corporate practices in the oil, gas, and mining industries, and supports the right of communities to participate meaningfully in decisions about the development of natural resources." [ $O x f a m$ aboga por políticas gubernamentales y prácticas corporativas en las industrias justas en los sectores del petróleo, gas y minería, y apoya el derecho de las comunidades de participar de forma significativa en la toma de decisiones sobre el desarrollo de los recursos naturales.»] Oxfam America, "Resource Rights," https://policypractice.oxfamamerica.org/work/resource-rights/. El Programa Global de Industrias Extractivas de Oxfam Internacional está activo en 30 países. Oxfam, "Extractive Industries Global Program” (map), http://eimap.oxfam.org/. Para obtener más información sobre la estrategia en extracciones de Oxfam, ver Oxfam's Oxfam International, Achieving Natural Resource Justice: Extractive Industries Global Program Strategic Plan 2016 - 2019, https://www.oxfamamerica.org/explore/research-publications/achieving-naturalresource-justice/

${ }^{7}$ Ver, por ejemplo, Oxfam, Mapping Risks to Future Government Petroleum Revenues in Kenya (Oxford, 2016), https://www.oxfam.org/sites/www.oxfam.org/files/file attachments/rr-mapping-risks-petroleum-revenueskenya-060516-en.pdf 
Foto: La plataforma petrolera Heritage de ExxonMobil en el Océano Pacífico (Glenn Beltz).

\section{OXFAM}

Oxfam es una confederación internacional de 19 organizaciones que trabajan juntas en más de 90 países, como parte de un movimiento global a favor del cambio, para construir un futuro libre de la injusticia que supone la pobreza. Para más información, escribir a cualquiera de las organizaciones o ir a www.oxfam.org.

Oxfam Alemania (www.oxfam.de)

Oxfam América (www.oxfamamerica.org)

Oxfam Australia (www.oxfam.org.au)

Oxfam Brasil (www.oxfam.org.br)

Oxfam-en-Bélgica (www.oxfamsol.be)

Oxfam Canadá (www.oxfam.ca)

Oxfam Francia (www.oxfamfrance.org)

Oxfam GB (www.oxfam.org.uk)

Oxfam Hong Kong (www.oxfam.org.hk)

Oxfam IBIS (Dinamarca) (www.oxfamibis.org/)
Oxfam India (www.oxfamindia.org)

Oxfam Intermón (España)

(www.oxfamintermon.org)

Oxfam Irlanda (www.oxfamireland.org)

Oxfam Italia (www.oxfamitalia.org)

Oxfam México (www.oxfammexico.org)

Oxfam New Zealand (www.oxfam.org.nz)

Oxfam Novib (Países Bajos) (www.oxfamnovib.nl)

Oxfam Quebec (www.oxfam.qc.ca)

Oxfam Sudáfrica (www.oxfam.org.za)

Observador: KEDV (Oxfam Turquía) 